\title{
Programación de riego en brócoli (Brassica oleracea L. cv. italica) en los Andes ecuatorianos
}

\author{
Irrigation scheduling in broccoli (Brassica oleracea L. cv. italica) \\ in the Ecuadorian Andes \\ David Risco 1, 2, *, Alberto Gutiérrez"1, Jesús Val ${ }^{3}$, Juan León ${ }^{2}$, \\ Azahara Díaz', Paúl Benalcázar², Henar Prieto ${ }^{4}$
}

\begin{abstract}
RESUMEN
El cultivo de brócoli requiere altas cantidades de agua de riego para conseguir una producción óptima. Sin embargo, la creciente escasez de recursos hídricos hace necesario implementar sistemas de riego eficientes para optimizar la eficacia en el uso del agua. El objetivo del estudio fue evaluar la respuesta del cultivo de dos híbridos de brócoli, Avenger (AV) y Domador (DOM) a tres dosis de riego, 50\% de reposición de la evaporación del tanque clase A (R50), 100\% de reposición (R100) y 150\% de reposición (R150). Estos ensayos se realizaron en dos épocas: de febrero a mayo y de julio a octubre de 2016. El diseño experimental fue factorial con cuatro bloques y seis tratamientos. El momento óptimo de riego se determinó utilizando una sonda FDR. Durante el primer ensayo no se observaron diferencias en la producción entre los distintos tratamientos de riego, debido a las intensas lluvias y a la menor evapotranspiración, pero si hubo diferencias de producción entre el híbrido Domador (23.6 t ha-1) y Avenger $\left(21.5\right.$ t ha- $\left.{ }^{-1}\right)$. En el segundo ensayo, la producción fue mayor en R150 (40.5 t ha- $\mathrm{a}^{-1}$ que en R100 (28.2 tha-1) y que en R50 (31.0 $\left.\mathrm{t} \mathrm{ha}^{-1}\right)$ lo que indica que en condiciones muy secas es necesario incrementar el aporte hídrico para satisfacer las necesidades del cultivo. Comparando ambos experimentos, la producción fue más alta y el área foliar menor en el segundo ensayo, lo que sugiere que el momento más adecuado para el cultivo de brócoli en los Andes ecuatorianos es la época de menores lluvias y mayor evapotranspiración.
\end{abstract}

Palabras clave: Avenger, Domador, eficiencia en el uso de agua de riego, producción, área foliar.

\begin{abstract}
Broccoli culture needs high amounts of irrigation water to achieve optimum yields, however, given the growing scarcity of water resources, it is necessary to design more efficient irrigation strategies to be applied. The objective of the study was to evaluate the response of two broccoli hybrids, Avenger (AV) and Domador (DOM), and three irrigation doses, 50\% replenishment of an evaporation class A pan (R50), 100\% replenishment (R100) and 150\% replenishment (R150). These trials were carried out in two periods: from February to May and from July to October 2016. The experiments were carried out in a factorial design with four blocks and six treatments. The optimum irrigation time was determined using a FDR probe. During the first trial, there were no differences in yield between the different irrigation treatments, due to the high rainfall and low evapotranspiration, but differences

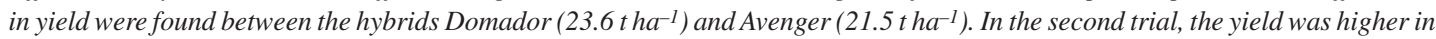

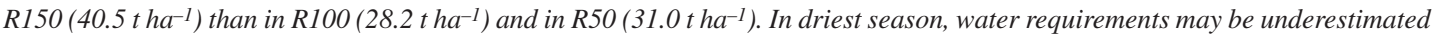
to supply the real necessities of the crop. Comparing both trials, the yield was higher and the leaf area smaller in the second trial, suggesting that the best time for broccoli cultivation in the Ecuadorian Andes is that time of lower rainfall and higher evaporation.

Key Words: Avenger, Domador, Irrigation water use efficiency, yield, leaf area.
\end{abstract}

1 Facultad de Ciencias Agropecuarias, Universidad Técnica de Ambato, Sector El Tambo-La Universidad, vía Quero, Cevallos, Tungurahua, Ecuador.

2 Facultad de Recursos Naturales, Escuela Superior de Chimborazo, Panamericana Sur km 1,5, Riobamba, Ecuador.

3 Departamento de Nutrición Vegetal. Estación Experimental de Aula Dei (EEAD-CSIC), Avda. Montañana 1005. 50059, Zaragoza, España.

4 Finca la Orden-Valdesequera (Junta de Extremadura), A5 km 372. 06187. Badajoz, España.

* Autor para correspondencia: da.risco@uta.edu.ec

Fecha de Recepción: 16 Agosto, 2017.

Fecha de Aceptación: 8 Diciembre, 2017. 


\section{Introducción}

El brócoli (Brassica oleracea L. cv. italica) es uno de los cultivos no tradicionales más importantes de los Andes ecuatorianos. El aumento en el cultivo, exportación y consumo que se ha producido en los últimos años se debe a su al alto valor nutricional, ya que contiene ácido ascórbico, vitamina $\mathrm{A}$, calcio y otros compuestos como flavonoides y glucosinolatos (Yoldas et al., 2008).

Por otra parte, el agua es un factor limitante en el cultivo de brócoli, siendo necesario un aporte de agua de riego para su correcto desarrollo. La importancia del agua de riego en la producción de brócoli está ampliamente documentada y se han descrito pautas para su manejo en diferentes países como Turquía (Erdem et al., 2010), Alemania (Gutezeit, 2004), Botswana (Imtiyaz et al., 2000), Estados Unidos (Johnson et al., 2016) o España (López-Urrea et al., 2009). Sin embargo, no se dispone de información suficiente acerca de las estrategias de riego para este cultivo en las condiciones edafoclimáticas de la sierra ecuatoriana.

El uso eficiente del agua de riego es un asunto de gran importancia debido principalmente a la competencia por el agua entre los agricultores, la industria y la población. Además, ante la previsible escasez de recursos hídricos como resultado del cambio climático es imperativo mejorar las prácticas de riego en la agricultura. El cálculo de la dosis de riego en cultivos hortícolas como el brócoli es necesario también para el adecuado desarrollo del cultivo, ya que un riego deficitario puede dar lugar a una reducción en la producción (Gutezeit, 2004). Para evitar el déficit hídrico, los agricultores normalmente aportan una cantidad de agua excesiva, pero este exceso puede producir el lixiviado de agua y nutrientes por debajo de la zona de absorción radicular, conduciendo también a un descenso en la producción (Imtiyaz et al., 2000).

La programación de riego es una de las herramientas para regular la cantidad de agua a aplicar en un cultivo que crece bajo condiciones de clima y suelo determinadas. Esta técnica consiste en aplicar agua a los cultivos en el tiempo y la cantidad necesarias para maximizar su producción y hacer un uso eficiente de este recurso. Las necesidades hídricas del cultivo se suelen calcular con la aproximación estándar de la FAO (Allen et al., 1998) que utiliza la evapotranspiración del cultivo (ETc) calculada como el producto de la evapotranspiración de referencia (ETo) y un coeficiente de cultivo $(\mathrm{Kc})$

$$
\mathrm{ETo}=\mathrm{ETc} \times \mathrm{Kc}
$$

El coeficiente de cultivo es un valor específico del cultivo y de su grado de desarrollo, y están disponibles los valores para diferentes tipos de cultivo (Allen et al., 1998). La evapotranspiración de referencia se puede calcular utilizando un tanque evaporímetro clase A, que es un método simple y utilizado ampliamente dada la facilidad de uso por los agricultores y la fácil transferencia de tecnología. La evaporación del tanque se relaciona con la evapotranspiración de referencia a través de un coeficiente:

$$
\mathrm{ETo}=\mathrm{K}_{\text {tanque }} \times \mathrm{E}_{\text {tanque }}
$$

Siendo $\mathrm{K}_{\text {tanque }}$ el coeficiente que se establece a través de parámetros climáticos como la humedad relativa y la velocidad del viento, y $\mathrm{E}_{\text {tanque }}$ la evaporación medida diariamente en el tanque que tiene unas medidas específicas.

Para determinar el momento más adecuado para la aplicación del riego se dispone de diferentes metodologías que permiten el registro del estado hídrico de la planta y del suelo. Los sensores volumétricos miden el contenido de humedad del suelo basándose en la capacitancia y se han hecho comunes en los últimos años debido al descenso en su coste y a la mejora de su eficacia, ya que permiten la toma continua de datos y una medición precisa del estado hídrico del suelo.

Con estos antecedentes, este trabajo se ha focalizado en la evaluación del efecto de diferentes niveles de riego sobre la producción, crecimiento vegetativo y eficiencia en el uso del agua de riego en el cultivo de dos híbridos de brócoli en la región de los Andes ecuatorianos.

\section{Materiales y Métodos}

El experimento fue llevado a cabo durante dos etapas, de febrero a mayo y de julio a octubre de 2016, en el Campus Querochaca de la Universidad Técnica de Ambato, Ecuador ( $01^{\circ} 22^{\prime} \mathrm{S}, 78^{\circ} 36^{\prime} \mathrm{W}$, $2865 \mathrm{msnm}$ ). El suelo de la zona del ensayo es un andisol típico, proveniente de cenizas volcánicas, con baja densidad aparente $\left(1,12 \mathrm{~g} \mathrm{~mL}^{-1}\right), \mathrm{pH}$ ligeramente ácido $(6,2)$, textura franco arenosa, 
capacidad de campo $14,1 \%$ y punto de marchitez permanente $7.6 \%$. El agua disponible para el perfil de $0-60 \mathrm{~cm}$ es de $43 \mathrm{~mm}$. El clima de la zona es ecuatorial de alta montaña (Pourrut et al., 1995), con una temperatura media de $12,8^{\circ} \mathrm{C}$, una precipitación media de $475 \mathrm{~mm}$ año ${ }^{-1}$ y una radiación de 1780 horas insolación. Las condiciones climáticas durante el experimento fueron registradas en una estación meteorológica situada a menos de $200 \mathrm{~m}$ del lugar del ensayo.

El diseño experimental fue factorial con dos factores (dosis de riego e híbrido) que contenían 6 tratamientos y cuatro repeticiones. Los tratamientos consistieron en tres niveles de reposición del tanque evaporímetro, $50 \%$ (R50), 100\% (R100) y $150 \%$ (R150) de la evaporación del tanque clase A, para facilitar la aplicación práctica. El material vegetal consistió en dos híbridos de brócoli, Avenger (AV) de grano fino y Domador (DOM) de grano fino a mediano. En función de la evaporación se hizo un cálculo semanal de la dosis de riego a aplicar. Los coeficientes de cultivo utilizados fueron 0,7 en la etapa inicial de crecimiento, de 0,7 a 1,05 durante el desarrollo vegetativo hasta alcanzar la cobertura completa, y de 1,05 a 0,95 durante la fase de emergencia de la pella (Allen et al, 1998). Se realizó un balance hídrico del suelo para conocer cuál fue el consumo del cultivo y, para verificar que los datos fueron correctos, se utilizó una sonda FDR (5TM, Decagon, Pullman, USA) situada en el tratamiento R100 a $30 \mathrm{~cm}$ de profundidad, en el punto medio entre dos goteros y próximo a la zona radicular de una planta. El límite de descenso tolerable considerado se situó por encima del punto de marchitez permanente. En ambos ensayos se realizó un riego de establecimiento del cultivo y los tratamientos de riego comenzaron dos semanas tras la siembra.

El primer ensayó se trasplantó el 8 de febrero y se recolectó el 4 de mayo, mientras que el segundo ensayo se trasplantó el 20 de julio y se recolectó el 25 de octubre. El marco de plantación fue de $0,4 \mathrm{~m} \times 0,4 \mathrm{~m}$ y cada planta fue regada por dos goteros con un caudal de $1,2 \mathrm{~L} \mathrm{~h}^{-1}$. La parcela experimental midió $15 \mathrm{~m}$ x 1,5 m con las plantas sembradas en disposición a tres bolillos cuando tenían un crecimiento fenológico de dos hojas. Todas las mediciones se realizaron en las plantas centrales de cada tratamiento.

El crecimiento vegetativo fue medido en cosecha en dos plantas por cada parcela experimental, tomando una fotografía de todas las hojas sobre un fondo blanco y estimando el área foliar mediante el programa Image J (https://imagej.nih.gov/ij/index. html). En esas mismas plantas se pesó la pella para realizar el cálculo de la relación entre el área foliar y la producción. La producción en cosecha se midió en 15 plantas por parcela experimental de la zona central en las que se pesó individualmente la pella y se midió el calibre. En ambos ensayos la cosecha se hizo en una sola pasada, cuando las pellas habían alcanzado la madurez comercial. La eficiencia en el uso del agua de riego (IWUE) se calculó como la producción de pella de brócoli total dividida entre el agua de riego aplicada estacional (Howell, 1990).

Los datos sobre los efectos de los tratamientos fueron analizados utilizando el programa Infostat (www.infostat.com.ar) mediante análisis de varianza. Para comparar los tratamientos se utilizó el test de Tukey.

\section{Resultados y Discusión}

Los datos climáticos variaron significativamente durante los dos ensayos. Entre febrero y mayo se registró una evapotranspiración de referencia acumulada de $198 \mathrm{~mm}$ y una precipitación total de $216 \mathrm{~mm}$, mientras que entre julio y octubre la evapotranspiración de referencia acumulada fue de $305 \mathrm{~mm}$ y la precipitación total de $106 \mathrm{~mm}$ (Figura 1). Este factor indujo que la producción general fuera superior en el segundo ensayo con respecto al primero (Tabla 2). A pesar de que las precipitaciones fueron menores, la evapotranspiración fue mayor, lo que influyó en que el desarrollo del cultivo fuera mejor en la segunda cosecha.

Las diferentes condiciones climáticas hicieron que la cantidad de agua aportada a los cultivos fuera diferente en los dos ensayos (Tabla 1). En el primero se aplicaron 10 riegos que acumularon un total de $30 \mathrm{~L} \mathrm{~m}^{-2}$ en el tratamiento R50, $56 \mathrm{~L} \mathrm{~m}^{-2}$ en R100 y $83 \mathrm{~L} \mathrm{~m}^{-2}$ en R150. En el segundo ensayo se aplicó una cantidad de agua mayor, realizando 12 riegos con un acumulado total de $49 \mathrm{~L} \mathrm{~m}^{-2}$ en $\mathrm{R} 50,97 \mathrm{~L} \mathrm{~m}^{-2}$ en $\mathrm{R} 100$ y $148 \mathrm{~L} \mathrm{~m}^{-2}$. La cantidad de agua aplicada en el cultivo de brócoli es muy diferente dependiendo de las condiciones edafoclimáticas y sistemas de riego que han sido descritas por los diferentes autores. Así, Johnson et al., (2016) en campos comerciales en Estados 


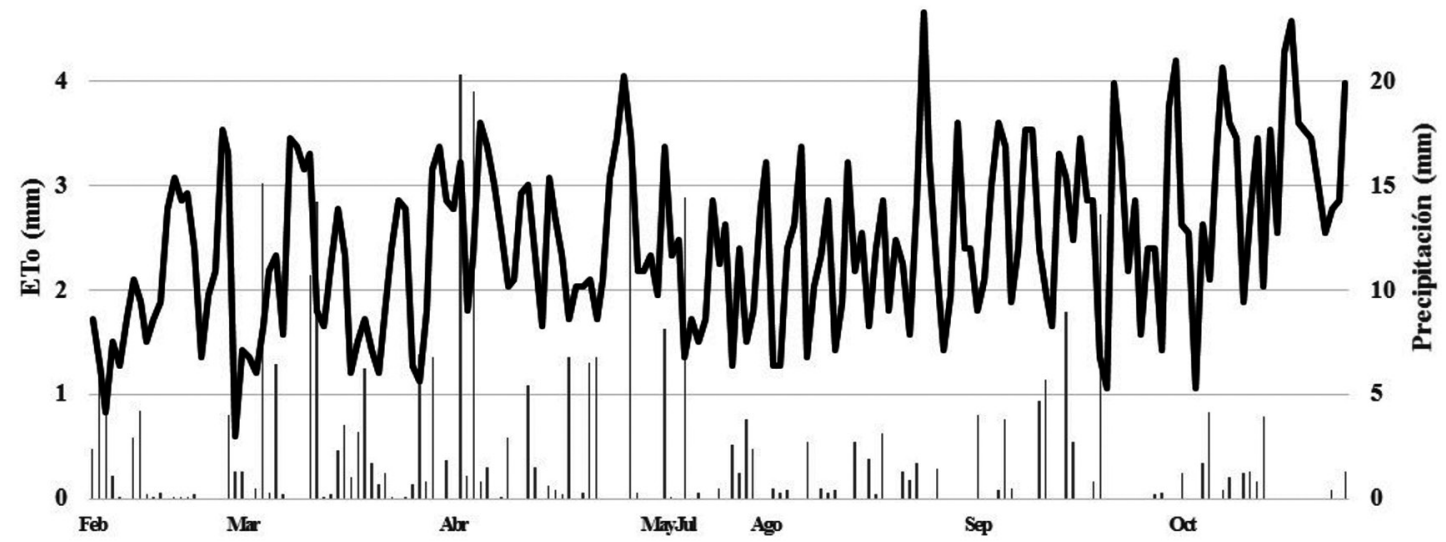

Figura 1. Condiciones climáticas durante el crecimiento de brócoli cv. Avenger y Domador en las épocas de febrero a mayo y de julio a octubre de 2016. Barras: Precipitación $(\mathrm{mm})$. Línea: Evapotranspiración de referencia $\left(\mathrm{mm} \mathrm{día} \mathrm{a}^{-1}\right)$ medida en un tanque evaporímetro clase A.

Tabla 1. Balance hídrico y riego aplicado $(\mathrm{mm})$ durante el crecimiento de brócoli c.v. Avenger y Domador en las épocas de febrero a mayo y de julio a octubre de 2016. ETo: Evapotranspiración de referencia; ETc: Evapotranspiración del cultivo.

\begin{tabular}{lcccccccr}
\hline & Feb & Mar & Abr & May & Jul & Ago & Sep & Oct \\
\cline { 2 - 9 } & $(8-29)$ & $(1-31)$ & $(1-30)$ & $(1-4)$ & $(20-31)$ & $(1-31)$ & $(1-30)$ & $(1-25)$ \\
\hline ETo (mm) & 44,3 & 66,3 & 77,2 & 10,1 & 23,8 & 98,3 & 106,2 & 76,3 \\
ETc (mm) & 34,2 & 58,1 & 79,3 & 9,8 & 18,8 & 73,7 & 79,7 & 77,5 \\
Precipitación (mm) & 27,9 & 86,0 & 93,5 & 8,2 & 25,2 & 65,9 & 81,2 & 17,3 \\
\hline \multicolumn{1}{c}{ Riego } & & & & & & & \\
\hline R50 & 7,5 & 9,8 & 8,8 & 3,8 & 2,5 & 14,5 & 18,8 & 17,6 \\
R100 & 11,3 & 19,5 & 17,2 & 7,5 & 2,5 & 29,9 & 38,1 & 35,4 \\
R150 & 15,3 & 29,3 & 26,7 & 11,3 & 2,5 & 48,4 & 58,4 & 53,2 \\
\hline
\end{tabular}

Unidos calcularon un promedio de $600 \mathrm{~mm}$ de agua aplicada; Erie et al., (1981) informaron de un uso del agua en Arizona (USA) de $500 \mathrm{~mm}$. En España, López-Urrea et al., (2009) aplicaron $249 \mathrm{~mm}$ en el agua de riego, mientras que Imtiyaz et al., (2000) usaron entre 150 y $375 \mathrm{~mm}$ en el híbrido Green Valiant en Botswana.

En cuanto a los parámetros de recolección, se observaron diferencias entre ambos ensayos, $\mathrm{y}$, dentro del mismo ensayo, respecto a la dosis de riego y al híbrido (Tabla 2). En el primer ensayo las producciones de todos los tratamientos de riego fueron similares, entre 22 y $24 \mathrm{t} \mathrm{ha}^{-1}$, aunque el calibre de las pellas fue superior en el tratamiento R150 con respecto a R50. El área foliar también fue similar en todos los tratamientos, pero el IWUE fue significativamente más alto en el tratamiento R50, permitiendo un uso más eficiente del agua, a costa de una ligera, aunque no significativa reducción de la producción que el resto de tratamientos. Bajo las condiciones lluviosas y de baja evapotranspiración de referencia del primer ensayo entre febrero y mayo, es posible reducir la dosis de riego sin provocar diferencias en la producción final. En cuanto a los híbridos, sí se observaron diferencias significativas. En el híbrido Domador se registraron producciones y calibres de pella superiores a las del híbrido Avenger, lo que podría indicar que Domador, un híbrido de grano mediano a grande, está mejor adaptado a la estación de mayores lluvias y menor evapotranspiración de referencia, condiciones que se dan en febrero en la sierra ecuatoriana.

Sin embargo, en el segundo ensayo, la producción y el área foliar fueron significativamente más altas en el tratamiento más regado (R150) 
Tabla 2. Parámetros de cosecha de los ensayos de febrero a mayo y julio a octubre de 2016. Los promedios en la misma columna compartiendo diferente letra son diferentes a p $<0,05$. R50: Riego hasta reposición del 50\% de la evaporación del tanque clase A; R100: Riego hasta reposición completa del tanque clase A; R150: riego hasta reposición del 150\% del tanque clase A; AV: Híbrido Avenger; DOM: Híbrido Domador; AF: Área foliar por planta; IWUE: Eficiencia en el uso del agua de riego; E.E.: Error estándar de la media.

\begin{tabular}{|c|c|c|c|c|c|c|}
\hline & Peso pella & Calibre & $\mathrm{AF}$ & AF / Producción & Producción & IWUE \\
\hline & $\mathrm{g}$ & $\mathrm{cm}$ & $\mathrm{m}^{2}$ & $\left(\mathrm{~m}^{2} \mathrm{~kg}^{-1}\right)$ & $\left(\mathrm{t} \mathrm{ha}^{-1}\right)$ & $\left(\mathrm{kg} \mathrm{m}^{-3}\right)$ \\
\hline \multicolumn{7}{|c|}{ Feb-May } \\
\hline $\mathrm{R} 50$ & $348,0 \mathrm{a}$ & $14,1 \mathrm{a}$ & $1,1 \mathrm{a}$ & $3,6 \mathrm{a}$ & $21,8 \mathrm{a}$ & $72,5 \mathrm{c}$ \\
\hline $\mathrm{R} 100$ & $351,3 \mathrm{a}$ & $14,5 \mathrm{ab}$ & $1,2 \mathrm{a}$ & $4,1 \mathrm{a}$ & $22,0 \mathrm{a}$ & $39,2 \mathrm{~b}$ \\
\hline $\mathrm{R} 150$ & $384,1 \mathrm{a}$ & $15,3 \mathrm{~b}$ & $1,2 \mathrm{a}$ & $3,2 \mathrm{a}$ & $24,0 \mathrm{a}$ & 28,9 a \\
\hline$p$-valor & 0,1 & 0,01 & 0,2 & 0,3 & 0,1 & $<0,0001$ \\
\hline E.E. & 13,0 & 0,3 & 0,1 & 0,4 & 0,8 & 1,6 \\
\hline $\mathrm{AV}$ & $344,7 \mathrm{a}$ & $14,1 \mathrm{a}$ & $1,21 \mathrm{a}$ & $4,0 \mathrm{a}$ & $21,5 \mathrm{a}$ & $45,1 \mathrm{a}$ \\
\hline DOM & $377,6 \mathrm{~b}$ & $15,1 \mathrm{~b}$ & $1,09 \mathrm{a}$ & $3,3 \mathrm{a}$ & $23,6 \mathrm{~b}$ & $48,7 \mathrm{a}$ \\
\hline$p$-valor & 0,03 & 0,001 & 0,09 & 0,2 & 0,03 & 0,06 \\
\hline E.E. & 10,6 & 0,2 & 0,1 & 0,4 & 0,7 & 1,3 \\
\hline \multicolumn{7}{|l|}{ Jul-Oct } \\
\hline $\mathrm{R} 50$ & $496,2 \mathrm{a}$ & $15,5 \mathrm{a}$ & $0,65 \mathrm{a}$ & $1,5 \mathrm{a}$ & $31,0 \mathrm{a}$ & $63,3 \mathrm{~b}$ \\
\hline R100 & $450,6 \mathrm{a}$ & $16,1 \mathrm{a}$ & $0,75 \mathrm{a}$ & $1,9 \mathrm{a}$ & $28,2 \mathrm{a}$ & 29,0 a \\
\hline $\mathrm{R} 150$ & $648,1 \mathrm{~b}$ & $17,1 \mathrm{a}$ & $1,04 \mathrm{~b}$ & $1,2 \mathrm{a}$ & $40,5 \mathrm{~b}$ & $27,4 \mathrm{a}$ \\
\hline$p$-valor & $<0,0001$ & 0,8 & 0,001 & 0,09 & $<0,0001$ & $<0,0001$ \\
\hline E.E. & 22,6 & 0,4 & 0,1 & 0,2 & 1,4 & 1,6 \\
\hline $\mathrm{AV}$ & $549,2 \mathrm{a}$ & $15,6 \mathrm{a}$ & $0,9 \mathrm{a}$ & $1,7 \mathrm{a}$ & $34,3 \mathrm{a}$ & $41,6 \mathrm{a}$ \\
\hline DOM & $514,0 \mathrm{a}$ & $16,9 \mathrm{a}$ & $0,8 \mathrm{a}$ & $1,3 \mathrm{a}$ & $32,1 \mathrm{a}$ & $38,2 \mathrm{a}$ \\
\hline$p$-valor & 0,2 & 0,2 & 0,1 & 0,2 & 0,2 & 0,1 \\
\hline E.E. & 18,4 & 0,7 & 0,1 & 0,2 & 1,2 & 1,3 \\
\hline
\end{tabular}

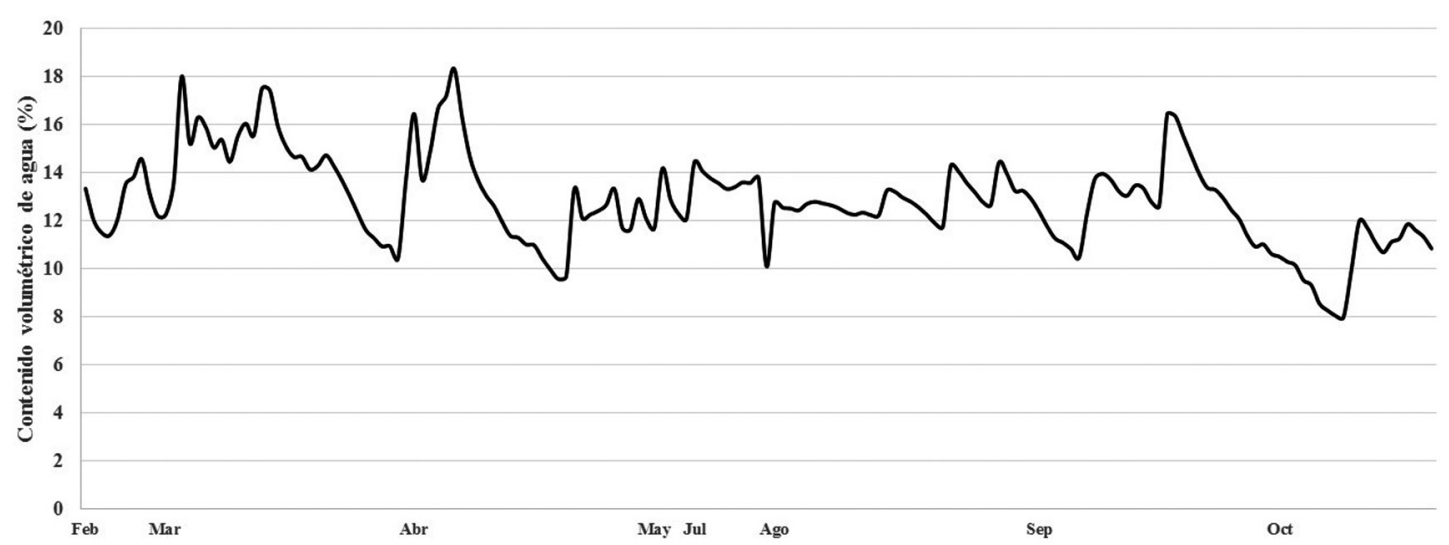

Figura 2. Contenido volumétrico de agua (\%) durante el crecimiento de brócoli cv. Avenger y Domador en las temporadas de febrero a mayo y de julio a octubre de 2016, medida con una sonda FDR situada en la parcela R100 a $30 \mathrm{~cm}$ de profundidad.

con respecto al resto (Tabla 2). Thompson et al., (2002) indicaron que los tratamientos de riego alto aumentaron los parámetros de cosecha en brócoli. Con las condiciones de menor lluvia y mayor evapotranspiración de referencia, fue necesario un aporte hídrico por encima de la reposición del $100 \%$ del tanque evaporímetro, para lograr una cosecha óptima. La eficiencia en el uso del agua de riego (IWUE) también fue mayor en el tratamiento menos regado (R50) aunque, a diferencia 
del primer ensayo, si se observó un descenso en la producción. Según Gutezeit, (2004), el riego por debajo de las necesidades hídricas puede conducir a un descenso en la producción. El comportamiento de los híbridos fue similar en cuanto a producción y crecimiento vegetativo, al contrario de lo que ocurrió en el primer ensayo.

El estudio comparado de la producción entre ambos ensayos demuestra que el rendimiento en el segundo fue significativamente más alto que en el primero, a pesar de haber aportado menos agua, si bien ésta se distribuyó de manera más homogénea y eficiente para el cultivo (Tabla 2). La diferencia en la producción entre ambos periodos puede ser atribuida a las diferentes condiciones climáticas lo que minimizó el efecto del riego sobre la producción de brócoli en el periodo de mayores lluvias y menores temperaturas, como ha sido observado previamente por diversos autores (Paschold et al., 2000; Erdem et al., 2010; Gutezeit, 2004). En cualquier caso, en ambos ensayos todas las producciones fueron superiores a las producciones medias registradas en la industria del brócoli en Estados Unidos, que se sitúa en 17,9 t ha ${ }^{-1}$ (Johnson et al., 2016). El área foliar, en cambio, fue superior en el primer ensayo con respecto al segundo. Mourão y Hadley, (1998), observaron aumentos en el área foliar de brócoli bajo condiciones de baja radiación solar. Esto sugiere que las condiciones climáticas y la distribución del riego también pueden afectar las relaciones fuente: sumidero de la planta y por tanto la producción final.

\section{Conclusiones}

En este trabajo se recogen datos útiles para el cultivo de brócoli en las condiciones agroclimáticas de los Andes ecuatorianos. Entre la información registrada se puede destacar que, en la estación de lluvias más copiosas, el incremento en la dosis de riego no produjo una producción más alta, por el contrario, la restricción de la dosis de riego no provocó reducciones significativas de la producción. En la estación de mayor evapotranspiración de referencia se precisó un aporte de agua de riego por encima de las necesidades hídricas teóricas del cultivo para alcanzar la mayor producción. En cuanto a los diferentes híbridos, Domador demostró ser más apto para el cultivo que Avenger en la estación más lluviosa y de menor evapotranspiración. Este estudio demuestra la necesidad de adaptar las estrategias de riego incluyendo herramientas y modelos de cálculo de evapotranspiración, además de la genética de las variedades y su adaptación a las condiciones edafoclimáticas de las distintas regiones.

\section{Agradecimientos}

Esta investigación fue realizada bajo el proyecto 1246 - CU - P - 2014 de la Universidad Técnica de Ambato (Ecuador). Los autores quieren agradecer a los operarios agrícolas del Campus Querochaca por su ayuda en los trabajos de campo, y a los técnicos del Instituto Nacional de Meteorología e Hidrología por su colaboración en la toma de datos climáticos.

\section{Literatura citada}

Allen, R.G.; Pereira, L.S.; Raes, D.; Smith, M.

1998. Crop evapotranspiration. Guide-lines for computing crop water requirements. FAO Irrigation and Drainage, 56. Rome, Italy.

Doorenbos, J.; Kassam, A.H.

1979. Yield response to water. FAO Irrigation and Drainage, 33. Rome, Italy.

Erdem, T.; Arin, L.; Erdem, Y.; Polat, S.; Deveci, M.; Okursoy,

H.; Gültas, H.T.

2010. Yield and quality response of drip irrigated broccoli (Brassica oleracea L. var. Italica) under different irrigation regimes, nitrogen applications and cultivation periods. Agricultural Water Management. 97: 681-688.

Erie, L.J.; French, O.F.; Brucks, D.A.; Harris, K.

1981. Consumptive use of water by major crops in the southwestern United States. USDA Cons. Res. Rep. Washington D. C. USA.
Gutezeit, B.

2004. Yield and nitrogen balance of broccoli at different soil moisture levels. Irrigation Science. 23: 21-27.

Howell, T.A.

1990. Water use by crops and vegetation. In: Stewart, B. A., and D. R. Nielsen. Irrigation of agricultural crops, agronomy. American Society of Agronomy, Madison.

Imtiyaz, M.; Mgadla, N.P.; Manase, S.K.; Chendo, K.; Mothobi. E.O. 2000. Yield and economic return of vegetable crops under variable irrigation. Irrigation Science. 19: 87-93.

Johnson, L.F.; Cahn, M.; Martin, F.; Melton, F.; Benzen, S.; Farrara, B.; Post, K.

2016. Evapotranspiration-based Irrigation Scheduling of Head Lettuce and Broccoli. HortScience. 51(7): 935-940.

López-Urrea, R.; Montoro, A.; López-Fuster, P.; Fereres, E. 2009. Evapotranspiration and responses to irrigation of broccoli. Agricultural Water Management. 96: 1155-1161. 
Mourão, I.M.G.; Hadley, P.

1998. Environmental control of plant growth development and yield in broccoli (Brassica oleracea L. var. Italica Plenk): crop responses to light regime. Acta Horticulturae. 459: 71-78.

Paschold, P.J.; Zengerle, K.H.; Kleber, J.

2000. Influence of irrigation on the yield and the nitrogen balance of broccoli. In: ISHS Acta Horticulturae 537: III International Symposium on Irrigation of Horticultural Crops, Lisbon, Portugal.

Pourrut, P.; Gómez, G.; Bermeo, A.; Segovia, A.

1995. Factores condicionantes de los regímenes climáticos e hidrológicos. En: Pourrut, P. Estudios de Geografía. El agua en el Ecuador. Clima, precipitaciones, escorrentía.
Colegio de Geógrafos del Ecuador. Corporación Editora Nacional. Quito, Ecuador.

Thompson, T.L.; Doerge, T.A.; Godin, R.E.

2002. Subsurface drip irrigation and fertigation of broccoli. I. Yield, quality and nitrogen uptake. Soil Science Society American Journal. 66: 186-192.

Verhaeghe, R.J.; van der Krogt, W.N.M.

1993. Crop water modelling with an operational management perspective. 15th International Congress of Irritation and Drainage. The Haughe, The Netherlands.

Yoldas, F.; Ceylan, S.; Yagmur, B.; Mordogan, N. 2008. Effects of nitrogen fertilizer on yield, quality and nutrient content in broccoli. Journal of Plant Nutrition. 31: 1333-1343. 
